\title{
The Relationship between Trabecular Bone Structure Modeling Methods and the Elastic Modulus as Calculated by FEM
}

\author{
Tomasz Topoliński, Artur Cichański, Adam Mazurkiewicz, and Krzysztof Nowicki \\ Faculty of Mechanical Engineering, University of Technology and Life Sciences, Kaliskiego 7 Street, 85-789 Bydgoszcz, Poland \\ Correspondence should be addressed to Adam Mazurkiewicz, adam.mazurkiewicz@utp.edu.pl
}

Received 25 November 2011; Accepted 19 January 2012

Academic Editors: A. Cömert, R. Pflugmacher, and E. Tanaka

\begin{abstract}
Copyright (C) 2012 Tomasz Topoliński et al. This is an open access article distributed under the Creative Commons Attribution License, which permits unrestricted use, distribution, and reproduction in any medium, provided the original work is properly cited.
\end{abstract}

\begin{abstract}
Trabecular bone cores were collected from the femoral head at the time of surgery (hip arthroplasty). Investigated were 42 specimens, from patients with osteoporosis and coxarthrosis. The cores were scanned used computer microtomography (microCT) system at an isotropic spatial resolution of 36 microns. Image stacks were converted to finite element models via a bone voxel-toelement algorithm. The apparent modulus was calculated based on the assumptions that for the elastic properties, $E=10 \mathrm{MPa}$ and $v=0.3$. The compressive deformation as calculated by finite elements (FE) analysis was $0.8 \%$. The models were coarsened to effectively change the resolution or voxel size (from 72 microns to 288 microns or from 72 microns to 1080 microns). The aim of our study is to determine how an increase in the distance between scans changes the elastic properties as calculated by FE models. We tried to find a border value voxel size at which the module values were possible to calculate. As the voxel size increased, the mean voxel volume increased and the FEA-derived apparent modulus decreased. The slope of voxel size versus modulus relationship correlated with several architectural indices of trabecular bone.
\end{abstract}

\section{Introduction}

Numerous papers have evaluated the mechanical properties of bone and have presented the opinion that these values can be determined not only by bone density but also by the properties of single trabeculae and the structure of the trabecular part of the bone [1-3]. In clinical practice, one of the most commonly applied methods of evaluating bone density is dual energy X-ray absorptiometry (DEXA). This method allows the determination of bone mineral density (BMD) and bone mineral content (BMC) as demonstrated by Hansson et al. [4], McBroom et al. [5], Beck et al. [6], and Cody et al. [7], which are used to indirectly determine fracture risk. Investigating density with DEXA is favorable because it is quick and the results are available immediately. However, the disadvantage of this method is that it does not consider the complexity of the structure of trabeculae.

To evaluate the structure of trabecular bone in vitro, computer microtomography (microCT) is commonly applied. This makes it possible to generate bone images with an accuracy of 6-8 microns. Based on images of the inner part of the bone obtained as a result of such investigations, micromechanical models of the bone structure subject to finite element (FE) calculations can be developed [8-11]. However, the application of this technique in vivo in clinical practice is difficult. The disadvantage of this technique is that it is time consuming, depending on the assumed performance accuracy. Currently, it is impossible to apply microCT to patients.

In clinical practice, it is possible to image trabecular bone using techniques such as multidetector computer tomography (MDCT) or high-resolution magnetic resonance imaging (HRMRI). The resolution of images obtained using these techniques is about $100-300$ microns at a slice density ranging from 300-500 microns [12-16]. At present, however, the availability of these techniques is limited.

Time limitations are one of the major problems of medical investigations because $\mathrm{X}$-ray investigation is harmful and requires the patient to stay motionless. In such cases, limiting the time of the medical investigation is beneficial for the patient, but this usually leads to a reduction in the resolution of the test. 
In numerous papers, the effects of increasing the voxel size of the FE model to a larger size than that obtained from microCT on the calculated values indices of the structure [17-20] and on the strength indices $[9,17,21-25]$ of trabecular bone have been studied. Kim et al. [25] found that for most of the structure indices, the results from analysis of images with larger voxel sizes, such as 110 microns, were correlated with results for a 21 microns voxel size. Cendre et al. [26] found that the HRCT (high-resolution microtomography) system with 150 microns resolution is not sufficient to predict the true values of the structural parameters measured by histomorphometry. Tabor [27] presents a new method of analysis called "optimal path" analysis of grey-level magnetic resonance imaging (MRI) images for improved estimation of the Young's modulus of trabecular bone samples.

The aim of our study is to determine how an increase in the distance between scans changes the elastic properties as calculated by FE models. The basis for the construction of our models is a set of scans of trabecular bone. Sample models were obtained from a microCT investigation. In our study, we assumed a simulated resolution change by altering the dimension of the voxel size in the direction of the axis of the sample. This simulation was based on the omission of some scans from the original data set in accordance with the proposed methodology.

The main advantage approach to modeling proposed by the authors is that models can be created based on a smaller microCT data set in comparison with modeling methods proposed by other authors. Consequently, in clinical practice, data collection will take less time and therefore will be limited dose of X-ray radiation absorbed by the patient.

This work also examines the relationship between the calculated modulus and the values of indicators describing the structure of bone.

\section{Material and Methods}

Cylindrical samples of $10 \mathrm{~mm}$ in diameter and $8.5 \mathrm{~mm}$ long were taken from the epiphyses of the heads of human femurs perpendicular to the axis of the neck, as shown in Figure 1 [28]. The study was approved by the Local Ethic Committee.

The heads were derived from the bones of patients after osteoporotic or coxarthrotic fractures and hip joint replacement surgery. The samples were investigated with a $\mu$ CT80 microtomograph (Scanco A.G., Switzerland) to obtain images of the inner part of the sample and to measure the selected indices of the structure: bone volume ratio (BV/TV), trabecular thickness (Tb.Th), and trabecular number (Tb.N) proposed by Parfitt et al. [29]. For each sample, an average of 230 microtomographic slices with a slice increment of 36 microns was obtained (parameters: $70 \mathrm{kV}, 114 \mu \mathrm{A}, 500$ projections $/ 180^{\circ}, 300 \mathrm{~ms}$ integration time). Measurements were made for 21 samples derived from bones diagnosed as osteoporotic (o) and for another 21 samples from bones diagnosed as coxarthrotic (c). For each sample, a basic geometric model was built as a comparative model.
Basic model development was based on a set of 230 binary images obtained using microCT that presented the structure of the sample in layers perpendicular to its axis. The size of the pixels in these images was $36 \times 36$ microns. The basic models were developed by creating voxels representing the bone, such that pixels on the same coordinates in both images had to be the color representing the bone.

The Hexahedral $(\mathrm{H})$ method was used as a reference, as described in the literature by Ulrich et al. [30]. Three additional different methods of model simplification were proposed, First-Last (F-L), First-Second (F-S), and FirstThird (F-T), all of which included limiting the number of scans. The main difference between these approaches was the method of using the scans for layer selection for voxel building.

2.1. The Hexahedral Method. In the first approach, fragments of the basic model in the shape of a cuberrille with a size equal to a multiple of 36 microns were analyzed for a range of side length of 72-288 microns.

Whenever more than $50 \%$ of the cuberrille volume was filled with voxels representing the bone (Figure 2(a)), a new voxel representing the bone was created with the dimensions of the cuberrille analyzed. In this approach, the basic model was built based on the original set of images obtained from microCT, and thus no data reduction (concerning the number of images) was needed to build the simplified model.

Simplification using the subsequent methods involved limiting the number of images used to build the model compared with the original number of images obtained with microCT.

2.2. The First-Last Method. To decrease the amount of data (the number of images) needed to build the model, the set of data obtained with microCT was limited. The difference, compared to the first approach, involved disregarding images of selected layers of the sample. In this model-simplification method, the voxel length $(d)$ was changed along the sample axis. This simplification involved building the model layer with two images of layers a multiple of 36 microns apart for a range of voxel length of 72-1080 microns. The condition for creating a voxel representing the bone was the same as for the basic model. The voxel created was 36 microns $\times 36$ microns $\times d$; the value $d$ was included in the range described above. The voxel structure diagram for this approach is given in Figure 2(b).

2.3. The First-Second Method. In the third approach, two successive images of layers obtained with microCT 36 microns apart were compared, after which a voxel of a predefined size " $d$ " was created. The value " $d$ " varied within the range of 72-1080 microns with a step size of 36 microns. This voxel development method is given in Figure 2(c).

2.4. The First-Third Method. In this approach, two images 72 microns apart were used. The voxel created was 36 microns $\times 36$ microns $\times d$, where the value of $d$ varied within the range 


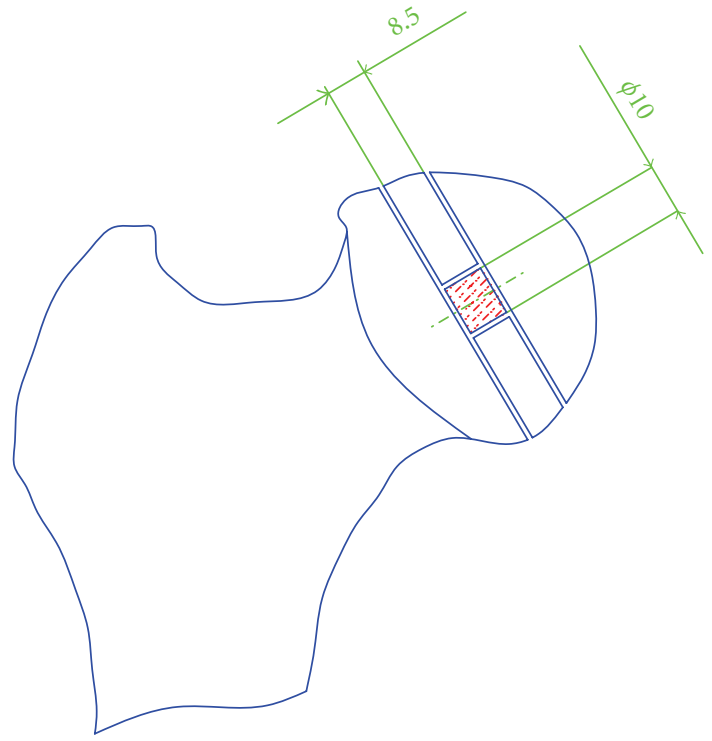

Figure 1: Trabecular bone sampling method. The sample axis coincided with the axis of the head and neck of the femur.

of 72-1080 microns with the step size of 72 microns. This voxel development method is given in Figure 2(d).

Numerical analyses were performed with the FE method using ANSYS Academic Research R12 (Ansys Inc., USA). The division network characteristic for the method used was prepared such that it constituted a direct transformation of the geometric notation of the structure considered in the "voxel-to-element" method [30]. The analyses used 3-D 8node SOLID45 elements with a side length of 36 microns. A single regular voxel (the first approach) corresponded to a single 3-D finite element. If the geometric model was built from prolonged cubicoid voxels (as in F-L, F-S and F-T methods), each voxel was replaced with multiple 3-D finite elements. Due to the stability of the process of iteration of the calculations when solving the numerical task from the division network, elements poorly connected with rest of the model and not affecting the rigidity of the structure were eliminated.

For the purpose of the analyses, which were linear, homogenous material properties $E=10 \mathrm{GPa}$ and $v=0.3$ were assumed [30]. Under such conditions, the results of the analyses reflected only the number and distribution of the respective cubes in the tissue-modeling structure.

In defining the boundary conditions in each approach, one base of the cylindrical sample was given zero displacement towards the axis. The opposite base was given a kinematic excitation acting along the axis of the cylinder to obtain the assumed compressive deformation $\varepsilon=0.8 \%$. The result of the calculations was the compressive axial force $(F)$ needed to obtain this assumed deformation. Based on these results, the elastic module $(E)$ was calculated.

Statistical analyses were performed with software R (The R Foundation for Statistical Computing).
TABLE 1: Values of structure indices obtained from microCT measurement.

\begin{tabular}{lcccc}
\hline Indices & Min-max & $\begin{array}{c}\text { Mean } \\
\text { value }\end{array}$ & SD & RSD, \% \\
\hline BV/TV, - & $0.068-0.392$ & 0.222 & 0.079 & 36 \\
Tb.Th, mm & $0.089-0.259$ & 0.151 & 0.036 & 24 \\
Tb.N, mm & $0.76-1.956$ & 1.436 & 0.267 & 19 \\
\hline
\end{tabular}

\section{Results}

Table 1 presents the range of values, mean value, standard deviation $(\mathrm{SD})$, and relative standard deviation $(\mathrm{RSD}=$ mean value/SD) of the selected indices of the sample structure obtained from microCT.

The results indicate a wide range of variation and large values of standard deviations. The mean volume $\left(V_{m}\right)$ of the samples, representing the bone of respective layers of the basic models, is given in Figure 3 according to increasing values. The curve of the $\mathrm{V}_{m}$ demonstrates three ranges. The selection was carried out using an independent description of the fragment ranges as indicated by a straight line using a linear regression method. The first range is dominated by the samples cut from the bone diagnosed as osteoporotic, and the third range is dominated by those diagnosed as coxarthrotic. It was observed that there are samples associated with a beginning, middle, and end of each of ranges. Hence, further analyses involving 9 samples, of which 5 were derived from osteoporotic bone and 4 from coxarthrotic bone, were necessary. Selected samples from each range are noted in Figure 3. For these samples, the calculations were done as an example, and the results of samples from the middle of each range were shown graphically.

For the basic models based on data from microCT, with voxels of 36 microns in size, the elastic modulus versus mean volume is presented in Figure 4. Interestingly, there is a strong relationship between these parameters, which confirms the possibility of performing full evaluations for selected samples. Figure 4 also shows selected samples marked according to Figure 3 for which the relationship between $E$ and $V_{m}$ has a high coefficient of determination $\left(R^{2}=0.96\right)$.

The Relative modulus (Relative $E$ ) was calculated, which is the elastic modulus obtained from the calculations for a given model relative to that obtained for the basic model (voxel size 36 microns). Graphical calculations are presented for three representative samples from each of the ranges investigated. The representative sample from the range of lowest volume is marked as $\mathrm{o} 34$, that from the middle range as 038 and that from the greatest-volume range as c24. Figure 5 presents the relative module calculated for models built for the same samples with successive simplification methods.

The coefficient of determinacy for the relative moduli determined for all of the samples and structural simplification 


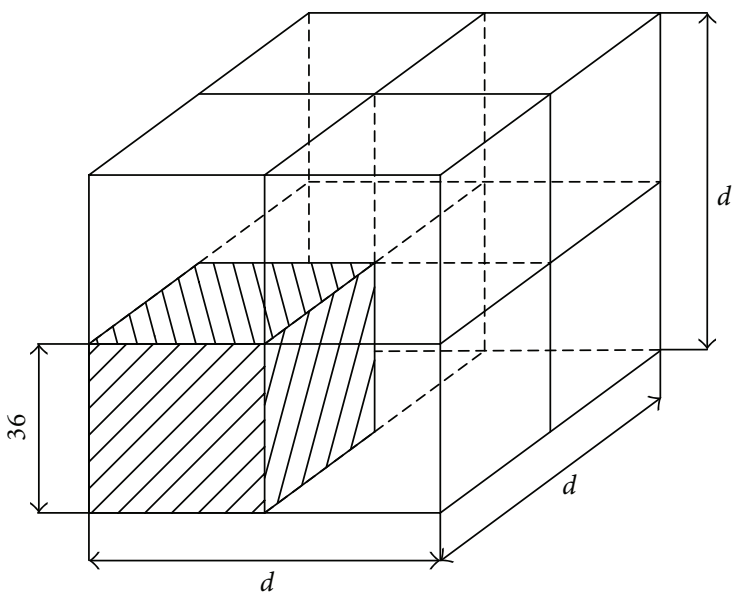

(a)

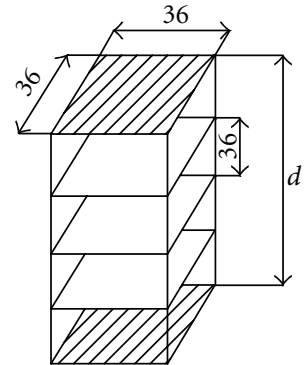

Image obtained from $\mu \mathrm{CT} 80$ that was excluded from analysis

Image obtained from $\mu \mathrm{CT} 80$ that was included in analysis

(b)

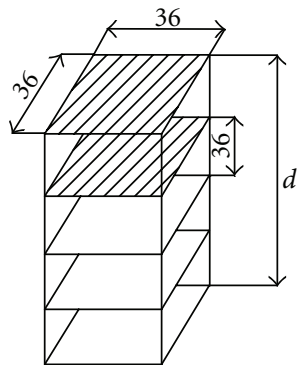

Image obtained from $\mu \mathrm{CT} 80$ that was excluded from analysis

Image obtained from $\mu \mathrm{CT} 80$ that was included in analysis

(c)

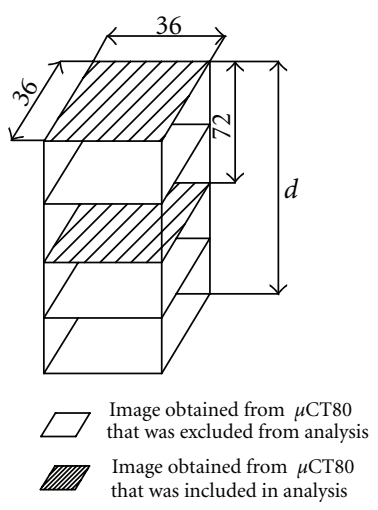

(d)

Figure 2: Voxel structure diagrams of length $d$ using the (a) Hexahedral, (b) First-Last, (c) First-Second, and (d) First-Third methods.

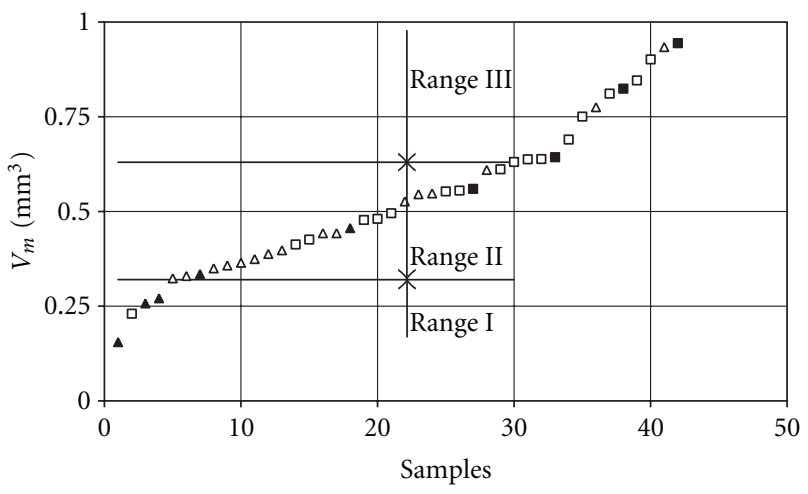

$\triangle$ Osteoporotic

a Coxarthrotic

FIgURE 3: Increasing values of $V_{m}$ for all samples.

methods considered (Figure 5) is high, ranging from $0.92-$ 0.99, which confirms a strong relationship between the modulus and the voxel length assumed for FE analyses.

Regression lines determined using the $\mathrm{H}$ method (Figure 5), the F-L layers method and structural simplification methods give relatively high absolute values of regression coefficients, except the results for the F-L method for sample c24. The range of effectiveness of these methods is, in practice, determined with voxels having a length in the range of 100-150 microns. Regression lines determined with the F-S method and the F-T method of structure simplification show almost threefold lower absolute values 


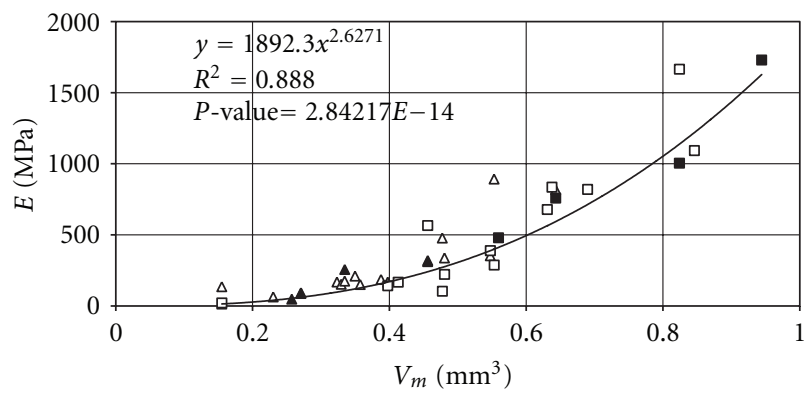

FIGURE 4: Dependence of the elastic modulus on the mean volume $V_{m}$.
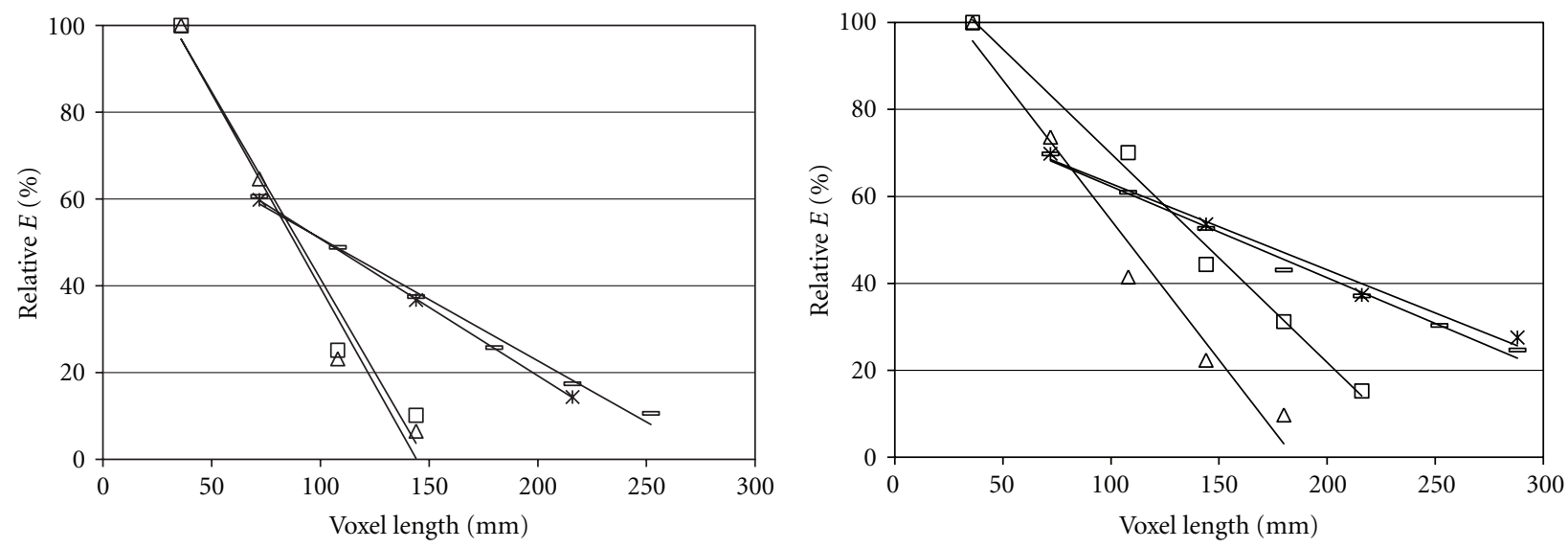

$\square \mathrm{H} \quad y=-0.0086 x+1.2782, R^{2}=0.9692 ; P=0.1124$

$\square \mathrm{H} \quad y=-0.0048 x+1.1796, R^{2}=0.9917 ; P=0.0003$

$\triangle$ F-L $y=-0.0089 x+1.2907, R^{2}=0.9746 ; P=0.096$

$\triangle$ F-L $y=-0.0064 x+1.1897, R^{2}=0.9743 ; P=0.0018$

- F-S $y=-0.0028 x+0.7924, R^{2}=0.9901 ; P=3.7 e-5$

- F-S $y=-0.0021 x+0.8336, R^{2}=0.992 ; P=1.2 e-5$

* F-T $y=-0.0032 x+0.8245, R^{2}=0.9999 ; P=0.0061$

* F-T $y=-0.002 x+0.8284 R^{2}=0.988 ; P=0.0007$

(a)

(b)

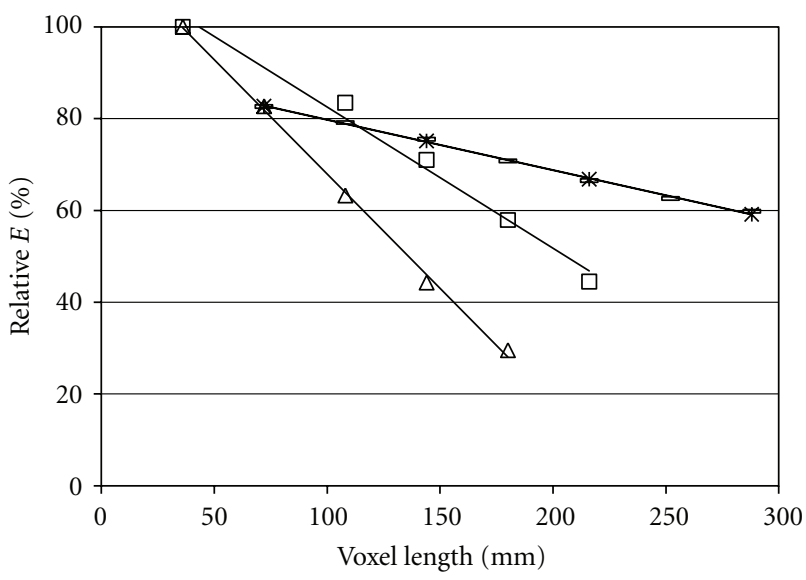

$\square \mathrm{H} \quad y=-0.0031 x+1.1378, R^{2}=0.9863 ; P=0.0007$

$\triangle$ F-L $y=-0.005 x+1.1775, R^{2}=0.998 ; P=3.6 e-5$

- F-S $y=-0.0011 x+0.9076, R^{2}=0.9967 ; P=1.7 e-6$

* F-T $y=-0.0011 x+0.907, R^{2}=0.9996 ; P=0.019$

(c)

Figure 5: The results for the three samples simplified by four different methods (a) o34, (b) o38, and (c) c24. 


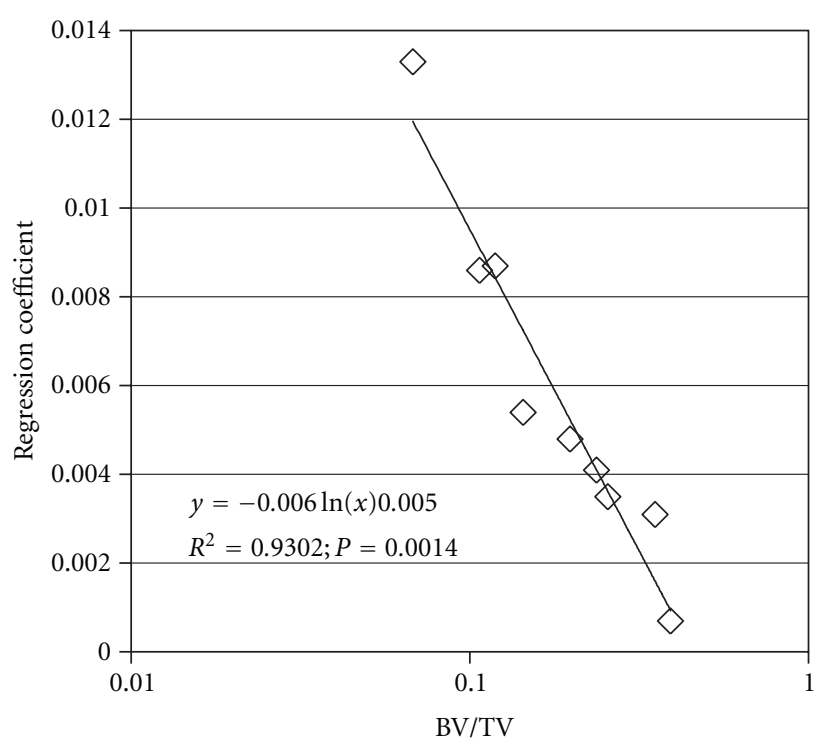

(a)

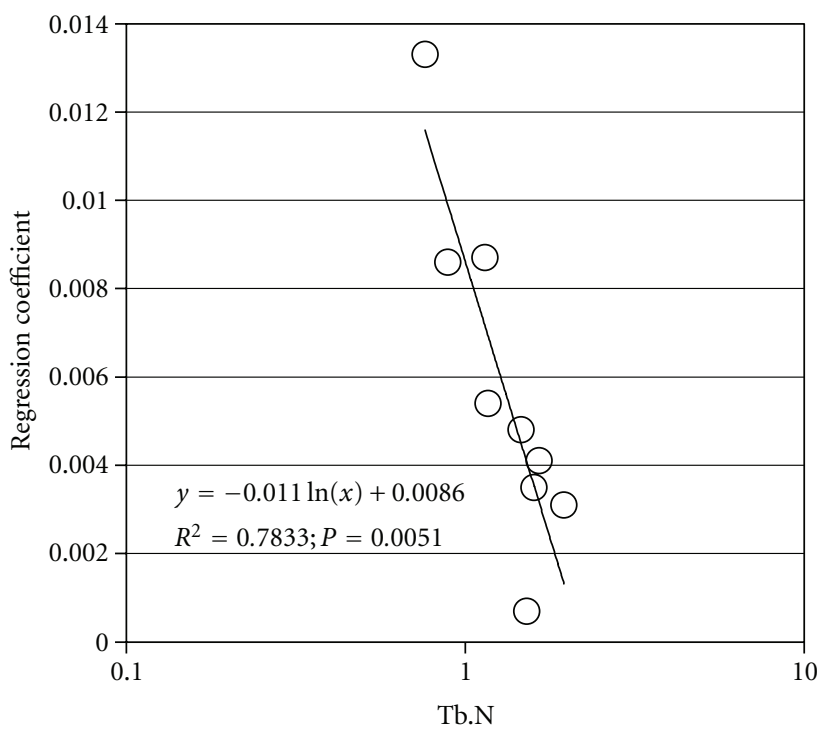

(c)

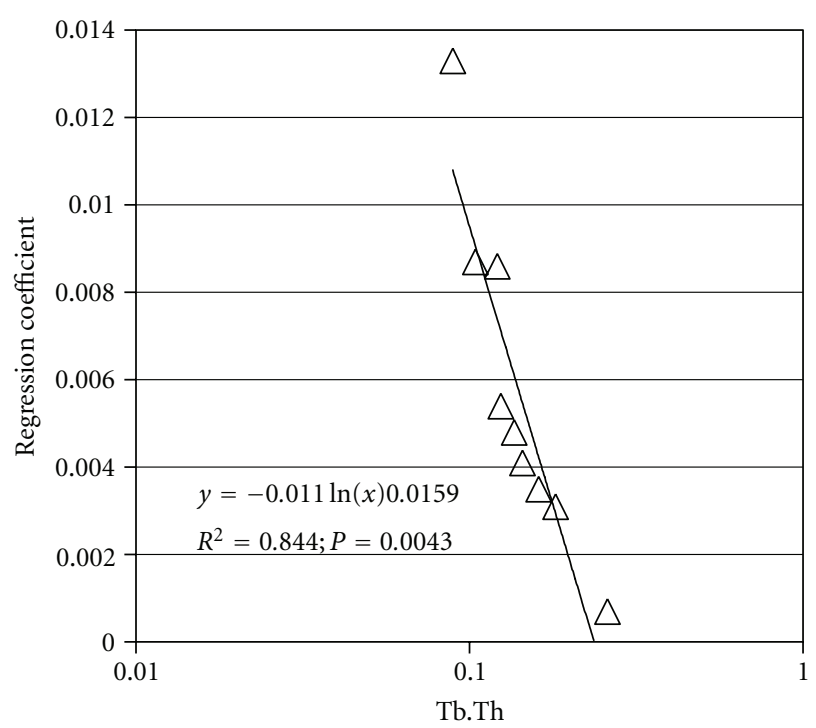

(b)

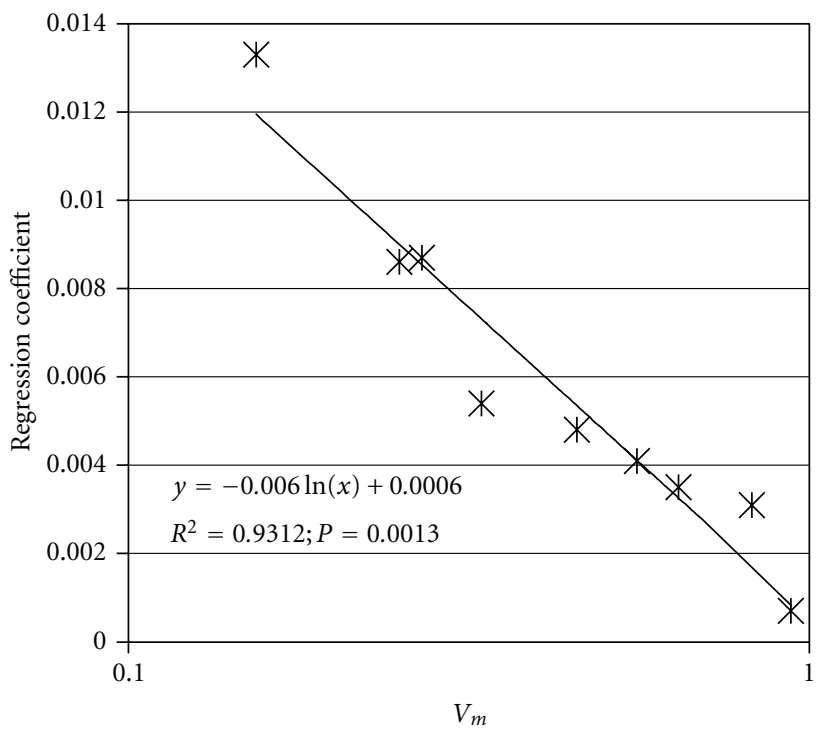

(d)

FIGURE 6: Relationships between the regression coefficients calculated with the hexahedral method of simplification and the structural indices (a) BV/TV, (b) Tb.Th, (c) Tb.N, and (d) $V_{m}$.

of regression coefficients compared with the previous two methods, outside of the c24 sample as well.

Based on a statistical test of the hypothesis conducted for equality of intercept and regression coefficient equality for the F-S and F-T methods, the value of $\alpha=0.005$ cannot be denied.

It is interesting that for larger values of $V_{m}$, that is, samples comprising higher levels of bone tissue, simplification of the structure has less influence on the calculated value of $E$. This is visible for the sample c24 for the F-S and F-T methods, where the value of Relative $E$ versus voxel length reaches $60 \%$.
The range of effectiveness of these methods is, in practice, determined with voxels having a length in the range of 200 250 microns.

Figure 6 presents the relationships between the regression coefficient calculated using one structural simplification method, the $\mathrm{H}$ method, and the selected structural indices. To plot these diagrams, the relative modulus for a voxel size up to 300 microns was calculated for each simplification method, and its logarithmic approximation was made. Then, the relationship between the absolute value of the regression coefficient and a given indices of the structure was established. 
TABLE 2: The relationship between the coefficients of determination (from the graphs RE versus voxel length) and structural indices of bone.

\begin{tabular}{lcccc}
\hline Method & Indicator & $a$ & $b$ & $R^{2}$ \\
\hline \multirow{3}{*}{ H } & BV/TV & -0.006 & -0.005 & 0.9302 \\
& Tb.Th & -0.011 & -0.0159 & 0.8440 \\
& Tb.N & -0.011 & 0.0086 & 0.7833 \\
& $V_{m}$ & -0.006 & 0.0006 & 0.9312 \\
\hline \multirow{3}{*}{ F-L } & BV/TV & -0.003 & 0.0004 & 0.8071 \\
& Tb.Th & -0.006 & -0.006 & 0.8126 \\
& Tb.N & -0.005 & 0.0076 & 0.6017 \\
& $V_{m}$ & -0.003 & 0.0034 & 0.8100 \\
\hline \multirow{3}{*}{ F-S } & BV/TV & -0.00008 & 0.0003 & 0.4414 \\
& Tb.Th & -0.002 & -0.0014 & 0.4981 \\
& Tb.N & -0.001 & 0.0019 & 0.2808 \\
& $V_{m}$ & -0.00007 & 0.001 & 0.4321 \\
\hline \multirow{3}{*}{ F-T } & BV/TV & -0.002 & -0.0019 & 0.7469 \\
& Tb.Th & -0.004 & -0.0057 & 0.6162 \\
& Tb.N & -0.004 & 0.0032 & 0.6935 \\
& $V_{m}$ & -0.002 & 0.0002 & 0.7406 \\
\hline
\end{tabular}

The relationships between the relative modules calculated using four structural simplification methods and the selected structural indices are given in Table 2. The diagrams, for all simplification methods, were created with the convention on Figure 6; the slope (a) and the intercept (b) of the regression lines were read. These values, together with the corresponding determination coefficients, $R^{2}$, are given in Table 2.

In the course of this analysis, we refer to the relationship between the regression coefficients (for the relative modulus) and the selected indices of the structure of the bone. For all of the structural simplification approaches analyzed, the relationship between BV/TV and $V_{m}$ was found to have the highest degree of occurrence. For the $\mathrm{H}$ and the F-L methods, achieved determination coefficients were up to 0.93 . For the F-S and the First-Third methods, determination coefficients of up to 0.74 were obtained.

\section{Discussion}

Our model of the bone structure involved the use of microtomographic scans 36 microns apart. This distance falls within the most frequently applied range, namely, from 6-8 to 60 microns [31], although recently, distances of 10 microns [32], 20 microns [33, 34], and 82 microns [35] have been commonly applied. The scanning resolution can significantly affect the structure modeled and thus influence the results of the calculations of mechanical properties [36].

In our work, we used 3-D 8-node hexagonal elements, as reported by Kosmopoulos et al. [37]; Bevill and Keaveny [34]; Mazurkiewicz and Topoliński [28]. In other studies, different types and dimensions of elements are used in analyses of the structure of bone. Four-node elements [38] and regular 2-D honeycomb [39] have been used in other research studies of trabecular bone. One report proposes a simplified approach to modeling the variation in trabeculae geometry with bar-and beam-type finite elements, with the analysis considering the variation in the direction, length and thickness of the trabeculae [40]. Similar considerations have been reported by other authors, who modeled each rod-like trabecula with one thickness-matched beam and each platelike trabecula with several beams [41]. Calculation using 1-D or 2-D elements can lead to excessive simplification of the model and reduce exactness of the calculations.

In the analyses performed in this paper, it was assumed that the trabecular bone tissue element is isotropic and linearly elastic $(E=10 \mathrm{GPa}, v=0.3)$, as reported by other authors $[27,37,42]$. We are aware that other approaches have been applied. For example, besides the standard approach, heterogeneous FE models have been proposed, for which the value of the tissue modulus was approximated from the degree of mineralization of bone (DMB) [43]. In the above heterogeneous FE model, calculations of the elastic modulus of human trabecular bone under axial compression are considered, which is a common load case in such calculations $[28,30,31]$.

In this paper, four methods of changing the voxel size of the bone model structure are considered. In the first method, the size of the voxel was changed at unchanged criteria of bone identification, and in the succeeding three, the simplification was carried out by limiting the number of scans. The latter cases can lead to similar calculation results using less data than the modulus calculations without simplifications. To build all of the models, we used binary images obtained after thresholding original data obtained from $\mu$ CT80. A threshold value was determined based on the settings of the devices.

In general, all of the calculation methods proposed lead to decreased values of the calculated modulus. As expected, the greater the simplification, the greater was the decrease in the modulus. The simplification of the structure following the Hexahedral method results in smaller decreases in the modulus. The results of the calculations using this method are in agreement with results presented by Bevill and Keaveny [34]. These other results which corroborate our studies performed calculations of mechanical properties of trabecular bone for vertebral, femoral neck, and greater trochanteric specimens. Calculations included the elastic modulus and the yield stress, based on data from $\mu \mathrm{CT} 20$ with 20 microns resolution. These quantities were calculated for voxel sizes of 40 microns, 80 microns, and 120 microns. In previous work [34], which corresponds to the first approach presented here, comparable results for the elastic modulus are reported for the range of $20-80$ microns.

Smaller decreases in the elastic modulus are obtained with the First-Second and First-Third methods. These results do not differ from each other, demonstrating that the simplification method assumed in these approaches does not cause a loss of essential information on the structure of the bone modeled. Performing further research with that method, such as searching for the maximum voxel length not 
causing significant changes in the elastic modulus, will affect the potential of using this research in vivo.

In each case, the decrease in the modulus increases with a greater degree of structural damage. In this paper, the $\mathrm{Vm}$ is used, and thus the lower the $\mathrm{Vm}$, the greater the decrease in the modulus. In the work [34] similar conclusions are reported for BV/TV. The decreases in the elastic modulus are a linear function of voxel size, and the coefficients of determinacy are above 0.96. An increase in voxel size is accompanied with a decreased module. This decrease is more significant for smaller values on BV/TV. Thus, inaccuracy in the calculation of the Young modulus is connected with the structure of the sample.

Application of the method of F-S and F-T allows for better representation of the strength of the bone compared to the $\mathrm{H}$ method. For F-L method, obtained results were less accurate than those compared to the $\mathrm{H}$ method.

The Hexahedral and First-Last methods are useful for a smaller voxel size in comparison to the First-Second and First-Third methods. In both cases, modulus correction should be done, depending on the structural bone parameters. If the bone sample is more porous, greater correction should be done.

For the Hexahedral and First-Last methods, there are strong relationships between the structure indices and the values of the regression coefficients (directional) obtained from the plots of $E$ versus voxel length for voxel size of 200250 microns. For the other methods, these relationships are satisfactory for the voxel length 100-150 microns.

In comparison to the First-Second method, the FirstThird method gives a better relationship between module and structural indices of bone, despite the higher distance between scans.

The statements above apply to all samples from both groups of bones. While better results are obtained for samples comprising higher levels of bone tissue.

\section{Acknowledgment}

This research was supported by the Polish State Committee for Scientific Research as part of research program N N501 308934.

\section{References}

[1] P. Ammann and R. Rizzoli, "Bone strength and its determinants," Osteoporosis International, vol. 14, supplement 3, pp. S13-S18, 2003.

[2] E. Lespessailles, C. Chappard, N. Bonnet, and C. L. Benhamou, "Imaging techniques for evaluating bone microarchitecture," Joint Bone Spine, vol. 73, no. 3, pp. 254-261, 2006.

[3] M. Matsubara, S. Morita, K. Shinomiya, R. Kawamata, K. Nakamura, and I. Kashima, "Structuring parameters for assessment of bone quality using a morphological filter and star volume analysis: structuring property in the cancellous bone of the human femoral head," Journal of Bone and Mineral Metabolism, vol. 21, no. 1, pp. 48-56, 2003.

[4] T. Hansson, B. Roos, and A. Nachemson, "The bone mineral content and ultimate compressive strength of lumbar vertebrae," Spine, vol. 5, no. 1, pp. 46-55, 1980.
[5] R. J. McBroom, W. C. Hayes, and W. T. Edwards, "Prediction of vertebral body compressive fracture using quantitative computed tomography," Journal of Bone and Joint SurgerySeries A, vol. 67, no. 8, pp. 1206-1214, 1985.

[6] T. J. Beck, C. B. Ruff, K. E. Warden, W. W. Scott, and G. U. Rao, "Predicting femoral neck strength from bone mineral data: a structural approach," Investigative Radiology, vol. 25, no. 1, pp. 6-18, 1990.

[7] D. D. Cody, S. A. Goldstein, M. J. Flynn, and E. B. Brown, "Correlations between vertebral regional bone mineral density (rBMD) and whole bone fracture load," Spine, vol. 16, no. 2, pp. 146-154, 1991.

[8] D. Ulrich, T. Hildebrand, B. van Rietbergen, R. Müller, and P. Rüegsegger, The Quality of Trabecular Bone Evaluated with Micro-Computed Tomography, FEA and Mechanical Testing, Bone Research in Biomechanics, IOS Press, 1997.

[9] A. J. C. Ladd, J. H. Kinney, D. L. Haupt, and S. A. Goldstein, "Finite-element modeling of trabecular bone: comparison with mechanical testing and determination of tissue modulus," Journal of Orthopaedic Research, vol. 16, no. 5, pp. 622628, 1998.

[10] R. Müller, H. van Campenhout, B. van Damme et al., "Morphometric analysis of human bone biopsies: a quantitative structural comparison of histological sections and microcomputed tomography," Bone, vol. 23, no. 1, pp. 59-66, 1998.

[11] T. E. Ciarelli, D. P. Fyhrie, M. B. Schaffler, and S. A. Goldstein, "Variations in three-dimensional cancellous bone architecture of the proximal femur in female hip fractures and in controls," Journal of Bone and Mineral Research, vol. 15, no. 1, pp. 32-40, 2000.

[12] S. Boutroy, M. L. Bouxsein, F. Munoz, and P. D. Delmas, "In vivo assessment of trabecular bone microarchitecture by highresolution peripheral quantitative computed tomography," Journal of Clinical Endocrinology and Metabolism, vol. 90, no. 12, pp. 6508-6515, 2005.

[13] M. Ito, K. Ikeda, M. Nishiguchi et al., "Multi-detector row CT imaging of vertebral microstructure for evaluation of fracture risk," Journal of Bone and Mineral Research, vol. 20, no. 10, pp. 1828-1836, 2005.

[14] C. Graeff, W. Timm, T. N. Nickelsen et al., "Monitoring teriparatide-associated changes in vertebral microstructure by high-resolution CT in vivo: results from the EUROFORS study," Journal of Bone and Mineral Research, vol. 22, no. 9, pp. 1426-1433, 2007.

[15] J. A. MacNeil and S. K. Boyd, "Accuracy of high-resolution peripheral quantitative computed tomography for measurement of bone quality," Medical Engineering and Physics, vol. 29, no. 10, pp. 1096-1105, 2007.

[16] A. Alberich-Bayarri, L. Marti-Bonmati, R. Sanz-Requena, E. Belloch, and D. Moratal, "In vivo trabecular bone morphologic and mechanical relationship using high-resolution 3-T MRI," American Journal of Roentgenology, vol. 191, no. 3, pp. 721-726, 2008.

[17] M. Kothari, T. M. Keaveny, J. C. Lin, D. C. Newitt, H. K. Genant, and S. Majumdar, "Impact of spatial resolution on the prediction of trabecular architecture parameters," Bone, vol. 22, no. 5, pp. 437-443, 1998.

[18] C. R. Jacobs, B. R. Davis, C. J. Rieger, J. J. Francis, M. Saad, and D. P. Fyhrie, "The impact of boundary conditions and mesh size on the accuracy of cancellous bone tissue modulus determination using large-scale finite-element modeling," Journal of Biomechanics, vol. 32, no. 11, pp. 1159-1164, 1999. 
[19] A. Laib and P. Rüegsegger, "Calibration of trabecular bone structure measurements of in vivo three-dimensional peripheral quantitative computed tomography with $28-\mu \mathrm{m}-$ resolution microcomputed tomography," Bone, vol. 24, no. 1, pp. 35-39, 1999.

[20] M. Ding and I. Hvid, "Quantification of age-related changes in the structure model type and trabecular thickness of human tibial cancellous bone," Bone, vol. 26, no. 3, pp. 291-295, 2000.

[21] O. T. Chavez and T. M. Keaveny, "High-resolution finite element models of trabecular bone: the dependence of tissue strains and apparent modulus on imaging resolution," American Society of Biomechanics, pp. 23-24, 1995.

[22] B. van Rietbergen, H. Weinans, R. Huiskes, and A. Odgaard, "A new method to determine trabecular bone elastic properties and loading using micromechanical finite-element models," Journal of Biomechanics, vol. 28, no. 1, pp. 69-81, 1995.

[23] J. Homminga, R. Huiskes, B. van Rietbergen, P. Rüegsegger, and $\mathrm{H}$. Weinans, "Introduction and evaluation of a gray-value voxel conversion technique," Journal of Biomechanics, vol. 34, no. 4, pp. 513-517, 2001.

[24] Y. N. Yeni and D. P. Fyhrie, "Finite element calculated uniaxial apparent stiffness is a consistent predictor of uniaxial apparent strength in human vertebral cancellous bone tested with different boundary conditions," Journal of Biomechanics, vol. 34, no. 12, pp. 1649-1654, 2001.

[25] D. G. Kim, G. T. Christopherson, X. N. Dong, D. P. Fyhrie, and Y. N. Yeni, "The effect of microcomputed tomography scanning and reconstruction voxel size on the accuracy of stereological measurements in human cancellous bone," Bone, vol. 35, no. 6, pp. 1375-1382, 2004.

[26] E. Cendre, D. Mitton, J. P. Roux et al., "High-resolution computed tomography for architectural characterization of human lumbar cancellous bone: relationships with histomorphometry and biomechanics," Osteoporosis International, vol. 10, no. 5, pp. 353-360, 1999.

[27] Z. Tabor, "Estimating structural properties of trabecular bone from gray-level low-resolution images," Medical Engineering and Physics, vol. 29, no. 1, pp. 110-119, 2007.

[28] A. Mazurkiewicz and T. Topoliński, "Relationships between structure, density and strength of human trabecular bone," Acta of Bioengineering and Biomechanics, vol. 11, no. 4, pp. 5561, 2009.

[29] A. M. Parfitt, M. K. Drezner, F. H. Glorieux et al., "Bone histomorphometry: standardization of nomenclature, symbols, and units. Report of the ASBMR Histomorphometry Nomenclature Committee," Journal of Bone and Mineral Research, vol. 2, no. 6, pp. 595-610, 1987.

[30] D. Ulrich, B. van Rietbergen, H. Weinans, and P. Rüegsegger, "Finite element analysis of trabecular bone structure: a comparison of image-based meshing techniques," Journal of Biomechanics, vol. 31, no. 12, pp. 1187-1192, 1998.

[31] H. K. Genant, C. Gordon, Y. Jiang, T. F. Lang, T. M. Link, and S. Majumdar, "Advanced imaging of bone macro and micro structure," Bone, vol. 25, no. 1, pp. 149-152, 1999.

[32] D. Arola, D. Bajaj, J. Ivancik, H. Majd, and D. Zhang, "Fatigue of biomaterials: hard tissues," International Journal of Fatigue, vol. 32, no. 9, pp. 1400-1412, 2010.

[33] L. Rapillard, M. Charlebois, and P. K. Zysset, "Compressive fatigue behavior of human vertebral trabecular bone," Journal of Biomechanics, vol. 39, no. 11, pp. 2133-2139, 2006.

[34] G. Bevill and T. M. Keaveny, "Trabecular bone strength predictions using finite element analysis of micro-scale images at limited spatial resolution," Bone, vol. 44, no. 4, pp. 579-584, 2009.
[35] N. Vilayphiou, S. Boutroy, E. Sornay-rendu et al., "Finite element analysis performed on radius and tibia HR-pQCT images and fragility fractures at all sites in postmenopausal women," Bone, vol. 46, no. 4, pp. 1030-1037, 2010.

[36] G. Diederichs, T. M. Link, M. Kentenich et al., "Assessment of trabecular bone structure of the calcaneus using multidetector CT: correlation with microCT and biomechanical testing," Bone, vol. 44, no. 5, pp. 976-983, 2009.

[37] V. Kosmopoulos, C. Schizas, and T. S. Keller, "Modeling the onset and propagation of trabecular bone microdamage during low-cycle fatigue," Journal of Biomechanics, vol. 41, no. 3, pp. 515-522, 2008.

[38] M. C. Michel, X. D. E. Guo, L. J. Gibson, T. A. McMahon, and W. C. Hayes, "Compressive fatigue behavior of bovine trabecular bone," Journal of Biomechanics, vol. 26, no. 4-5, pp. 453-463, 1993.

[39] M. Taylor, J. Cotton, and P. Zioupos, "Finite element simulation of the fatigue behaviour of cancellous bone," Meccanica, vol. 37, no. 4-5, pp. 419-429, 2002.

[40] Z. Miller and M. B. Fuchs, "Effect of trabecular curvature on the stiffness of trabecular bone," Journal of Biomechanics, vol. 38, no. 9, pp. 1855-1864, 2005.

[41] G. H. van Lenthe, M. Stauber, and R. Müller, "Specimenspecific beam models for fast and accurate prediction of human trabecular bone mechanical properties," Bone, vol. 39, no. 6, pp. 1182-1189, 2006.

[42] P. G. Coelho, P. R. Fernandes, H. C. Rodrigues, J. B. Cardoso, and J. M. Guedes, "Numerical modeling of bone tissue adaptation-A hierarchical approach for bone apparent density and trabecular structure," Journal of Biomechanics, vol. 42, no. 7, pp. 830-837, 2009.

[43] G. A.P. Renders, L. Mulder, G. E.J. Langenbach, L. J. van Ruijven, and T. M.G.J. van Eijden, "Biomechanical effect of mineral heterogeneity in trabecular bone," Journal of Biomechanics, vol. 41, no. 13, pp. 2793-2798, 2008. 


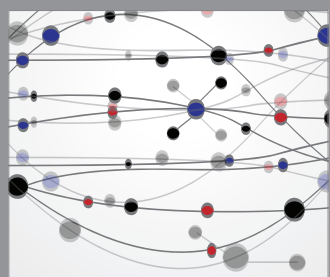

The Scientific World Journal
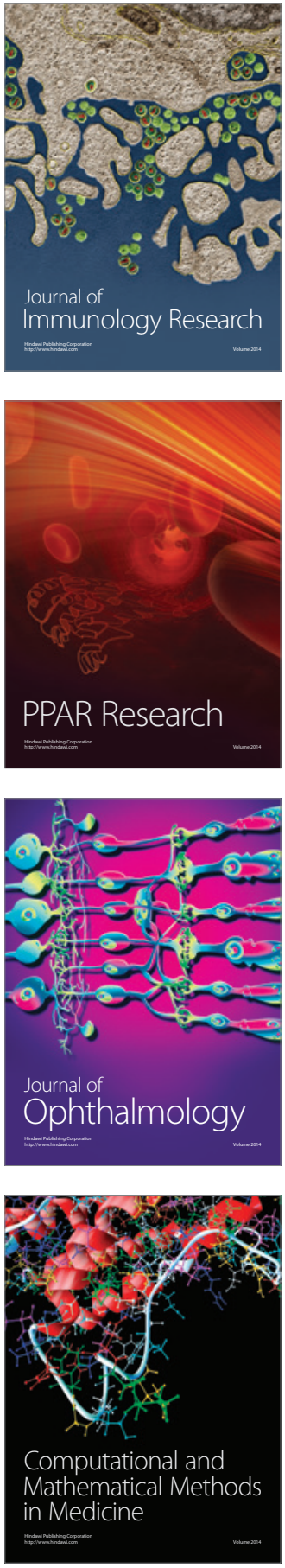

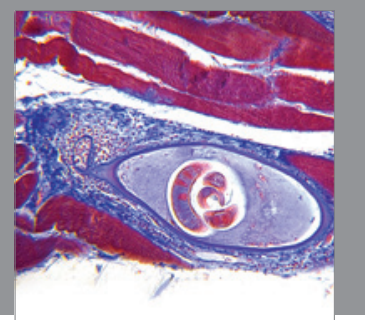

Gastroenterology

Research and Practice
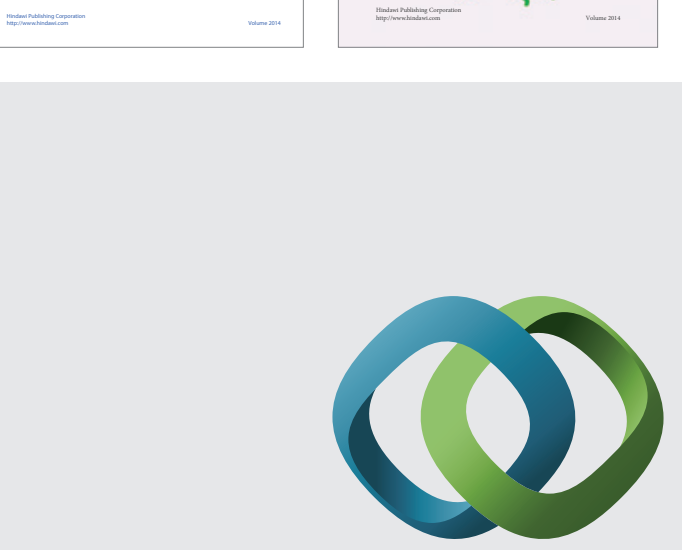

\section{Hindawi}

Submit your manuscripts at

http://www.hindawi.com
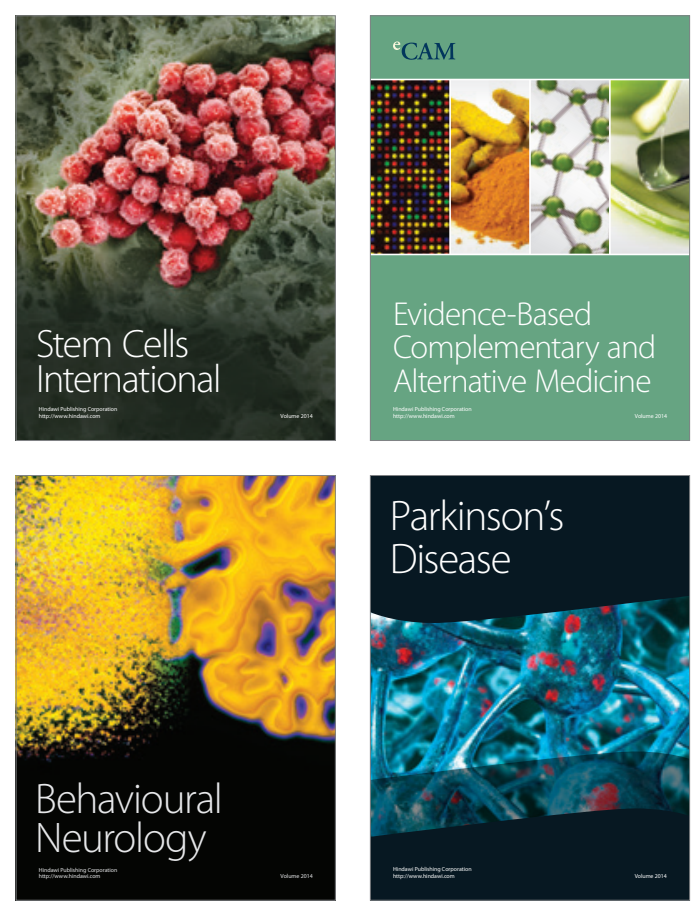

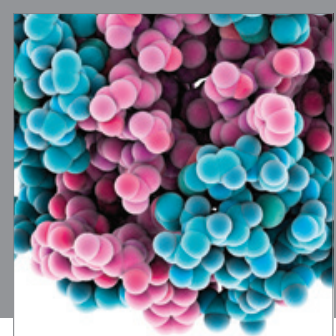

Journal of
Diabetes Research

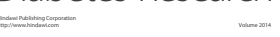

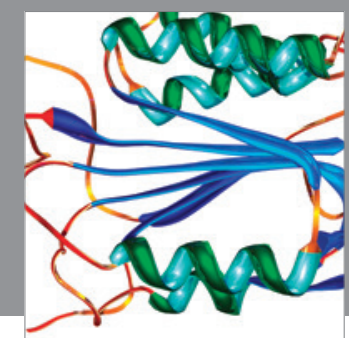

Disease Markers
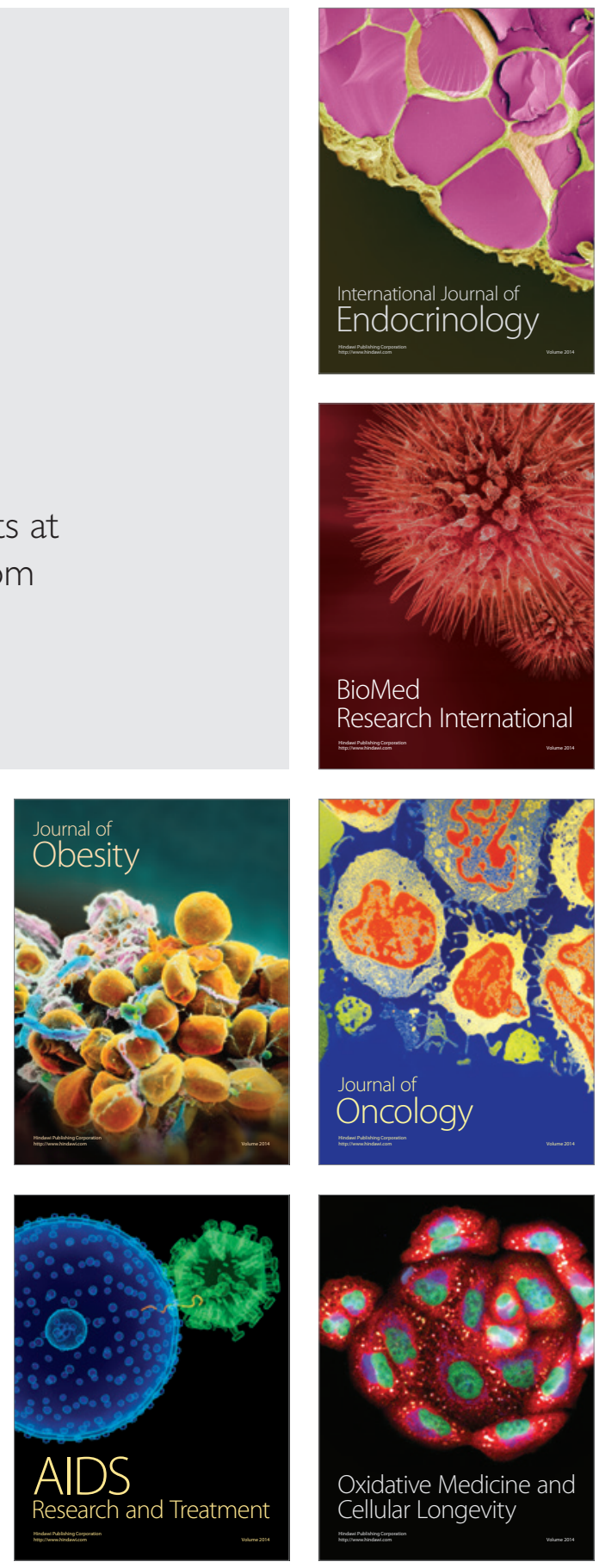